Voix et Images

\title{
Gilbert La Rocque : correspondance avec Murielle Ross (1963-1965). Extraits
}

\section{Donald Smith}

Volume 15, numéro 3 (45), printemps 1990

Gilbert La Rocque

URI : https://id.erudit.org/iderudit/200852ar

DOI : https://doi.org/10.7202/200852ar

Aller au sommaire du numéro

Éditeur(s)

Université du Québec à Montréal

ISSN

0318-9201 (imprimé)

1705-933X (numérique)

Découvrir la revue

Citer ce document

Smith, D. (1990). Gilbert La Rocque : correspondance avec Murielle Ross (1963-1965). Extraits. Voix et Images, 15(3), 328-351.

https://doi.org/10.7202/200852ar d'utilisation que vous pouvez consulter en ligne.

https://apropos.erudit.org/fr/usagers/politique-dutilisation/ 


\section{Gilbert La Rocque: correspondance avec Murielle Ross (1963-1965). Extraits*}

\section{présentée par Donald Smith}

Montréal-Nord, 1963. Gilbert La Rocque vient d'avoir vingt ans. Il sait déjà qu'il veut être écrivain, ou peut-être peintre. Cette année sera primordiale dans l'évolution du futur romancier, car il commence une correspondance avec Murielle Ross, jeune fille originaire de la Gaspésie, destinée à devenir la femme de Gilbert, après avoir été complice d'écriture, une oreille attentive et indispensable. Et quelle correspondance! Quelles lettres d'amour! On y découvre un La Rocque peu connu... galant, romantique, gracieux, méditatif, réservé; un La Rocque d'une autre époque, fin XVIII ${ }^{e}$ siècle sans doute, alors que naissait la sensibilité romantique, que les délires de l'imagination et les délices du sentiment captivaient les esprits; un La Rocque humoriste (voir, par exemple, la lettre du 7 mars 1964 où il est question des délicieux symptômes de la grippe); un La Rocque philosophe, énonçant maximes et pensées, fruits de sa réflexion.

Paradoxalement, bien que Gilbert et Murielle habitaient tous les deux à Montréal et se voyaient presque tous les jours, c'est par la poste que le jeune Gilbert, tout intériorisé et timide, a décidé de dialoguer avec la femme de sa vie! Tous les soirs, à minuit moins cinq, Gilbert La Rocque déposait Murielle à l'hôpital du Sacré-Cœur, à Cartierville, où elle travaillait comme infirmière auxiliaire. Ensuite, il rentrait chez lui et se livrait à un dialogue-monologue avec elle. C'est ainsi qu'a commencé un jeu épistolier qui a duré plus de deux ans.

J'ai compris, en lisant ces lettres réfractaires à tout matérialisme, jusqu'à quel point l'auteur des Masques était un homme profondément mal à l'aise dans notre monde capitaliste. À l'âge de vingt ans, Gilbert était " courtois ", affichant ce qu'il appelle, dans sa lettre du 7 janvier 1964, des accents volontairement antiques.

Les lettres d'amour de Gilbert La Rocque font découvrir un écrivain dans la vingtaine qui cherche sa voie, qui jouit du pouvoir évocateur des

* L'ensemble de cette correspondance, ainsi que tous les manuscrits inédits de Gilbert La Rocque, peuvent être consultés à la Bibliothèque nationale du Québec. 
mots, expliquant à sa bien-aimée l'importance de l'art dans le monde d'aujourd'hui, décrivant avec sensibilité des paysages intérieurs et extérieurs. Tant de monts hallucinants, de couleurs vives, chatoyantes; tant de toiles peintes par Gilbert La Rocque (dont celle décrite dans la lettre du 5 novembre 1963, tableau préféré de l'auteur, offert à Murielle en gage d'amour, ou celle, plus onirique et tourmentée, qui orne la page couverture de mon essai Gilbert La Rocque. L'écriture du rêve); tant de décors imprégnés de la personnalité 'de l'auteur, de ses extases et de ses tourments; tant d'arbres amaigris aux bras crispés; tant d'oiseaux, signes d'une tendresse à peine tangible... Qui, mieux que Gilbert La Rocque, possède cette aptitude à rêver sur la page blanche, à se déplacer à l'intérieur et à l'extérieur de paysages avec une voix hallucinée? Voilà des paysages fermentés il y a plus de vingt ans, destinés dès lors à réapparaitre dans les romans et dans la vie de Gilbert La Rocque, dont la dernière demeure fut la région envoûtante du mont Saint-Hilaire.

Dans ces lettres écrites avec beaucoup de soin, l'on constate avec émerveillement la puissance d'évocation spontanée de l'écriture de La Rocque, l'organisation instinctive de phrases rythmées, berceuses, hautement musicales. On trouve dans cette correspondance la même perfection de l'écriture que dans celle que Gilbert a entretenue avec Gérard Bessette, sauf que... bien sûr, le ton y est à l'opposé: avec Bessette, tout est grivois, scatologique, ironique, caricatural, gargantuesque, hyperbolique; avec Murielle, l'atmosphère est tendre, romantique, affable, pudique, beaucoup plus près de la litote que du grossissement stylistique. Mais saviez-vous que Gilbert La Rocque était un homme tendre, un séducteur courtois et respectueux? Murielle La Rocque le sait, et nous la remercions d'avoir eu la gentillesse et la perspicacité d'avoir offert ces lettres d'amour au grand public, au nom de la littérature, au nom d'une certaine vision du monde si chère à Gilbert La Rocque.

Dans ses lettres, Gilbert La Rocque livre des réflexions pertinentes sur le rôle de l'écriture dans le monde technologique. Il eût été pourtant si facile de tout simplement téléphoner à Murielle. Mais dans une lettre, Gilbert pouvait se laisser aller à la magie des mots, se lancer dans l'aventure de l'imaginaire, afin de vaincre les heures. La nécessité de l'écriture, même sous forme de lettres, guidait Gilbert La Rocque. Il encourageait Murielle à lui répondre; il écrivait régulièrement à son ami intime, Michel de Lorimier. Gilbert La Rocque voulait que tout le monde fasse l'expérience valorisante de l'écriture. Ceux et celles qui ont eu la chance de travailler avec lui chez Québec/Amérique en ont été les témoins privilégiés. Gilbert La Rocque a conclu un pacte d'amour avec les mots, ainsi qu'avec quelques amis. À l'écriture et aux amis, il est toujours resté fidèle, viscéralement loyal, tel un chevalier né à la mauvaise époque.

Les lettres d'amour de Gilbert La Rocque intéresseront sûrement les lecteurs de l'œuvre du romancier disparu, mais je sais aussi, les ayant fait lire à des gens peu acquis à la lecture romanesque, qu'elles 
enthousiasmeront toute personne intriguée à l'idée de se retrouver dans la peau d'un jeune homme fou d'amour et d'écriture. Et ceci est aussi vrai pour les hommes que pour les femmes, car le jeune épistolier excelle à rendre la présence de Murielle plus que réelle, puisque à la fois intérieure et anecdotique, imaginée et vécue, et à faire sortir le romantisme tapi au fond de chacun et de chacune.

Les vingt-deux lettres reproduites ici nous aident à comprendre la façon qu'avait Gilbert La Rocque de concevoir l'écriture. Une lettre, tout comme un roman, doit être dense, chargée, dépourvue de superflu. Gilbert La Rocque écrivait avec une retenue et une économie de mots exemplaires. Le monde irréel $d u$ rêve et la magie $d u$ songe constituent son inspiration première. $\grave{A}$ peine Gilbert La Rocque effleure-t-il le réel qu'aussitôt il le "déréalise", par le rêve. Écrire, c'est affronter la frontière abominable qui sépare le rêve du réel (lettre du $1^{\mathrm{er}}$ septembre 1964).

Romantique à l'âge de vingt ans, tourmenté par le poids de l'existence vers la fin de sa vie, Gilbert La Rocque n'a jamais changé quant à ses rapports avec les mots: il croyait à leur valeur suggestive et sensorielle. [...] ce poème demande que l'on aille au-delà des mots; audelà même des idées. Pour bien en saisir toute la portée, il faut lui laisser suggérer librement tout un monde de sentiments, tout le contenu humain qui lui est propre. Lis-le comme tu respirerais le parfum des fleurs [...] (lettre du 11 février 1964).

Pour s'initier à la littérature, Gilbert La Rocque lisait Lamartine, appréciant surtout la spontanéité et la sincérité, le chant intérieur qui s'élève de la musique des vers de l'auteur du «Lac». Les résonances intimes de Sully Prudhomme le fascinaient, mais il faut surtout évoquer Gérard de Nerval, dont les recherches sur les correspondances entre le rêve et la vie semblent avoir beaucoup influencé Gilbert La Rocque. Heureux prisonniers des mots, Gérard de Nerval et Gilbert La Rocque ont tous deux vécu plus du côté des songes que du réel. Le rêve d'absolu de Gilbert La Rocque n'est pas sans ressembler aux aspirations des grands romantiques, tel Villiers de l'Isle-Adam se réfugiant dans son monde intérieur, exaltant le pouvoir presque surnaturel des mots et de l'amour. Comme Victor Hugo, Gilbert La Rocque était tenté par l'occultisme, à l'affût du mystère des êtres et des choses. Dans ses lettres, il offre même son analyse de la calligraphie de Murielle Ross. Je sais que La Rocque croyait à ce qu'il qualifiait de supranaturel. En témoigne cette ombrelle de papier balançant sur un compas, placée dans un bocal, que Gilbert a réussi à faire tourner devant moi, en se concentrant, que par la force de sa volonté.

Musique, peinture et poésie étaient inséparables dans la vision littéraire de La Rocque. Pour lui, tout était musique et image. Combien de fois, dans ses lettres, se dit-il hypnotisé par une symphonie, modelant et rythmant ses phrases sur les impulsions de Bruckner, de 
Mahler ou de Wagner. Le jeune Gilbert explique dans le détail sa philosophie poétique fondée sur les correspondances mystérieuses [...] entre les formes, les couleurs, la musique, la pensée... (lettre du 2 juin 1964).

Sous l'influence de l'amour grandiose que Gilbert La Rocque éprouvait pour Murielle Ross, l'univers onirique du futur écrivain n'était pas encore troublé par les tourments et les déchirures violentes qui allaient caractériser ses six romans. La hantise de la mort, l'apparition progressive de cauchemars et les responsabilités du quotidien viendront bouleverser la perception idyllique de la réalité du jeune Gilbert de vingt ans. Alors que la mémoire enjolivait tout pour l'auteur des lettres d'amour, la force terrible de la mémoire profonde viendra perturber l'existence du romancier. Nous apprenons néanmoins dans ces lettres que La Rocque était, dès un jeune âge, tourmenté par l'imminence de la mort. Sa santé le préoccupait au plus haut point. Fumeur invétéré, il implorait Murielle de l'aider à se débarrasser de cette habitude destructrice. Des visions de corps décomposés hantaient l'écrivain. Et pourtant, le 11 janvier 1965, il exprimait un certain optimisme qui ne l'a peut-être jamais quitté: l'Esprit ne meurt pas. Parlait-il alors de sa propre mort? Sentait-il qu'il ne vivrait pas longtemps? Une lecture en profondeur du Passager semble le confirmer.

Même si les lettres d'amour révèlent un Gilbert La Rocque plus romantique que l'écrivain des années 70 et 80 , on peut quand même reconnaître les caractéristiques prédominantes du La Rocque adulte. Je suis de ceux qui ne tolèrent pas de demi-mesures, dit-il le 20 janvier 1964 . Ce que je hais, je le rejette au complet; ce que j'aime, je l'accepte tout entier, poursuit-il. Nous reconnaissons ici le polémiste qui, en tant qu'éditeur et auteur, s'en prenait avec force aux hypocrisies dans le monde du livre.

Dès l'âge de six ans, Gilbert La Rocque était un véritable rat de bibliothèque. Lorsqu'il a commencé à rédiger ses lettres d'amour, il était déjà sûr de ses moyens: certains [de mes poèmes] ont de grandes richesses, la beauté et la gloire (lettre du 10 février 1964). Gilbert La Rocque fuyait les entrevues; il n'aimait pas remettre verbalement les gens à leur place, préférant le mot écrit au contact réel. Lire ses lettres d'amour, c'est mieux comprendre sa personnalité complexe, sa retenue verbale, sa prédilection pour les mots: Je t'ai parlé [...] de la difficulté que jéprouve à extérioriser vocalement mes sentiments profonds. Chacun a une faiblesse à l'armure de sa personnalité:je t’ai révélé la mienne (9 mars 1964).

Gilbert La Rocque croyait à l'amour: amour des mots, amour des êtres qui lui étaient chers, amour de la nature, de son pays. Le refus de l'hypocrisie et de la déloyauté l'a toujours guidé:

Je vois par ma fenêtre le jour qui décline doucement et qui jette son pastel dans la verdure du jardin. Jamais le printemps ne m'a semblé si beau, si doux; jamais je n'ai ressenti comme à présent la joie qui se dégage de cette saison. C'est que l'amour a placé devant mes yeux un filtre merveilleux, un filtre à 
travers lequel tout prend la couleur du rêve. C'est notre printemps à nous seuls, car nous l'avons créé bien avant sa venue matérielle. (Lettre du 12 mai 1964)

Les lettres d'amour de Gilbert La Rocque racontent l'histoire d'une écriture et d'un amour naissants. L'un n'allait pas sans l'autre. Lorsque Gilbert a écrit sa première lettre, il connaissait Murielle depuis trois semaines.

\section{CORRESPONDANCE $*$}

Montréal-Nord, le 15 sept. 1963

Chère Murielle,

Venant de moi, une lettre peut te paraître quelque chose de bizarre, étant donné l'existence dù téléphone... Une lettre qui ne dit presque rien, qui n'annonce aucune nouvelle, mais qui va à l'aventure, apportant avec elle de quoi rompre un peu la routine quotidienne.

I faut que je t'avoue qu'en ce moment, malgré ce que je disais récemment, je fume des pyramides de cigarettes. Tant pis pour mes poumons!

Je veux que mon message soit court; mais je veux qu'il soit complet. Il ne le sera que dans la mesure où il contiendra une portion d'infini. Et comment les mots peuvent-ils exprimer ce qui appartient au monde irréel du rêve, à la magie du songe? Seule la poésie nous jette une passerelle pour nous permettre d'accéder à cet univers mystérieux.

J'ai écrit, il y a quelques jours, un poème que je vais prendre la liberté de te transcrire ici. Puis-je te rappeler que la poésie ne doit pas être lue par l'esprit mais bien par l'âme?... ce qui revient à dire qu'il ne faut pas trop chercher à comprendre: on n'a qu'à sentir en soi la musique se dégageant de la phrase, le choc transmis par l'image.

\section{Vers l'aube}

Le fantoche fiévreux

Au bal du sommeil

Je lui crie mon nom

Toute une vie est là

J'écris dans le sable

Une plainte éperdue

Je la grave mon sang

Sur un front ascendant

Ô mon sang pour mes yeux

L'écharpe enroulée

De linceul extatique

Le pantin de mes nuits

Les ficelles sont des murs

Et je redis mon nom

* Toute reproduction interdite. 
Cette fois pour toi seule

Món nom blême au chevet

De mon espoir cristal

À l'ouragan multiple

Résonnant la clairière

Toi seule désormais

Au sommet chaviré

De tout ce qui scintille

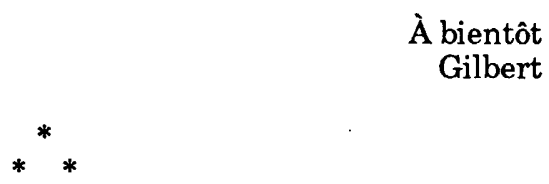

Montréal-Nord, le 24 sepț. 1963

Chère Murielle,

Voici venu le moment d'une autre rencontre que je vais tâcher de soustraire aux influences du temps et de l'espace. Considère cette lettre comme une sorte de poème en prose...

La nuit frappe à une fenêtre - et j'écris - et nous voici lancés contre un horizon incliné. Aux cheveux fous de la tèmpête rénovée, une trêve de la solitude parmi la foule. J'inscris au roc de l'oubli un nom qui renaît à chaque pas, à chaque fleur bleue des ténèbres. Des mousses d'ombre transparente viennent s'accrocher à nos rires - car nous avons vaincu les heures. Dans notre course folle, nous avons franchi la' frontière du rêve. Au plomb de l'orage qui s'exaspère, nous jetons parfois des bouffées de silence. Le silence que nous inventons en faveur du moindre bruit.

Voici la mer qui nous habite

Et voici l'or pâli du ciel

Et le feuillage de l'aurore

Voici les perles du matin

Un matin que personne d'autre ne peut apercevoir - car il n'existe qué par la magie du songe. Tout s'efface lentement dans un brouillard de soie incertaine. Tout disparaît et tout se réfugie sous des paupières closes...

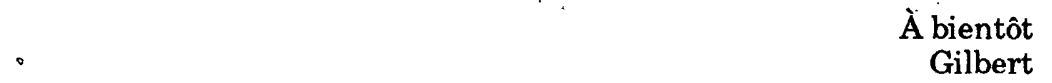

P.S. J'espère que tu as toujours, comme moi, le tabac en horreur...

Montréal-Nord, le 7 oct. 1963

Chère Murielle,

Je vais tout d'abord te raconter une courte anecdote qui apportera, je l'espère, une sorte de preuve qui puisse te convaincre de l'existence d'un monde supranaturel. 
Monsieur X avait envoyé son cuisinier prendre quelques jours de repos dans sa famille. Comme il détestait la solitude, $X$ recevait tous les soirs quelques amis avec qui il organisait des parties de bridge qui ne finissaient que tard dans la nuit. Le lendemain du départ du cuisinier, les invités arrivèrent comme à l'accoutumée et tout se passa normalement. Vers deux heures du matin, cependant, une sorte d'inquiétude, un vague malaise s'empara soudainement du petit groupe. Puis, tout à coup, les lumières s'éteignirent et tous purent entendre distinctement un grand bruit venant des cuisines. Monsieur X, ayant allumé une bougie, descendit avec ses hôtes dont le trouble augmentait sans cesse. Un spectacle des plus singuliers s'offrit à leurs yeux quand ils pénétrèrent dans la fameuse pièce: tout ce que les armoires avait contenu de casseroles, de chaudrons, d'ustensiles, tout gisait pêle-mêle au milieu du plancher. Cette scène qui n'avait pourtant rien d'effrayant en soi provoqua chez Monsieur X un choc tel qu'on dut le transporter jusqu'à sa chambre.

Le lendemain, on apprit qu'à l'heure même où l'événement se déroulait, le cuisinier était terrassé par une crise cardiaque et expirait dans les bras de ses parents.

Les témoins étaient dignes de foi et je crois que le fait se passe de commentaires...

Tu pourras m'en reparler si tu désires avoir de plus amples explications.

Je désire aussi t'entretenir un peu d'un aspect de ma poésie; un aspect qui te regarde personnellement. Dans un poème, l'élément qui doit prédominer, selon moi, est l'image symbolique. Dans cet ordre d'idées, si je te choisis, toi, comme sujet d'inspiration, je ne parlerai pas directement de ce que tu es, mais plutôt de quelque chose qui peut te symboliser, si la chose est possible. Je vais te donner un exemple, mais sois sûre que l'image est bien au-dessous de la réalité...

\section{Impression}

Sous la garde de l'image

Dénouée autour de moi

À son cil que l'ombre ment

L'ombre bleue qui bat au vent

Sous le signe des cheveux

Par le mystère du soir

Le silence d'un parfum

Enveloppe une présence.

À très bientôt

Gilbert

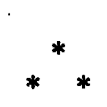

Montréal-Nord, le 5 novembre 1963

Chère Murielle,

Jai devant moi un tableau. II représente un paysage assez singulier. On y voit un lac dont les eaux ont pris des teintes violettes pour célébrer la fin d'un 
jour d'automne. Au loin, barrant un horizon incendié, quelques vagues montagnes occupent la droite de cette peinture: presque tout le mystère qui se dégage du panorama provient justement de ces monts, aux ombrages indécis et hallucinants. Au premier plan, on peut apercevoir deux bouleaux que le reste du décor fait ressortir d'une façon telle qu'on pourrait les croire réels. Le plus grand est dénudé et ses bras amaigris se crispent sur un ciel un peu verdâtre; l'autre porte un feuillage assez abondant où le vert se mêle au jaune et au roux.

Dans l'ensemble, le tableau ne comporte pas de couleurs qui frappent l'œil de façon éclatante. Plus qu'une image, j’ai voulu en l'exécutant qu'il devienne moi-même en l'imprégnant de toute ma personnalité. Si je me permets de vouloir te l'offrir, il y a une raison. Pour faire une œuvre qui vaille quelque chose, il faut que je me trouve dans un état d'esprit tout particulier. Et, à ce point de vue, je pense que c'est la mieux réussie de mes toiles que tu vas posséder. Celle à laquelle je tiens le plus aussi: mais n'est-il pas naturel que je te destine ce que j'ai de meilleur? De la sorte, si le don est indigne de toi, du moins te sera-t-il présenté avec les meilleures intentions du monde.

Je dois terminer ici cette lettre pour ne pas manquer la dernière levée du courrier : je tiens à ce que tu la reçoives mercredi, tel que promis...

\section{À bientôt}

Gilbert

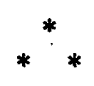

Montréal-Nord, le 19 nov. 1963

Chère Murielle,

Je veux que cette lettre soit simple; qu'elle fixe dans le temps un moment d'arrêt, juste le temps de nous trouver ensemble, si je puis m'exprimer ainsi. Une feuille de papier qui cherche à faire galoper les heures d'une longue semaine.

Je t'ai souvent décrit des paysages étranges, une nature singulière. Toi qui as vécu une partie de ta jeunesse dans un des coins les plus pittoresques de notre pays, tu as eu sous les yeux de magnifiques tableaux champêtres: je le sais pour avoir déjà parcouru (assez vite, il est vrai) la Gaspésie. Mais je suis sûr que tu seras d'accord avec moi si je dis ceci: les scènes d'autrefois que tu peux revoir dans ta mémoire te paraissent plus admirables que lorsque tu avais l'occasion de les contempler en réalité. C'est un fait que la mémoire tend à enjoliver ce qu'elle nous présente. Et c'est un bienfait que de pouvoir se souvenir dans de telles conditions.

Mais le contraire existe aussi; là où la réalité dépasse de beaucoup tous les artifices de l'esprit. Par exemple, il me semble évident qu'aucun prodige de l'imagination et de la mémoire ne puisse remplacer la présence véritable d'une personne qui nous est chère. Le souvenir qu'on garde de cette même personne n'apporte comme résul tat qu'une hâte de plus en plus grande de la revoir: un autre bienfait de la mémoire.

Pour finir, je cite deux vers d'un grand poète qui dit la mệme chose de façon différente: 
Un seul être vous manque

Et tout est dépeuplé

(Lamartine)

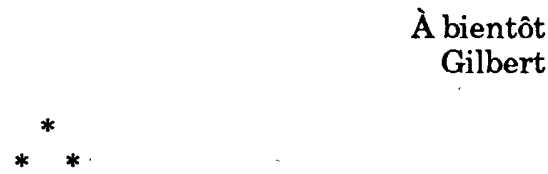

Montréal-Nord, le 7 janvier 1964

Chère Murielle,

La vie normale reprenant son cours, je puis t'écrire ma lettre-poème traditionnelle. Je crois t'avoir déjà affirmé que le but de ces petits messages était de nous soustraire, ne fût-ce que quelques minutes, au monde réel. Mais, dans le fond, je pense que ces lettres peuvent avoir une grande utilité, car elles facilitent entre nous une compréhension de plus en plus grande. Je crois profondément qu'un poème sorti du plus profond du cœur expose à découvert la personnalité, la façon de penser, l'âme de celui qui l'a composé. Et, d'autre part, même si la chose peut te sembler étrange, le seul fait de créer pour toi un poème m'aide à mieux te comprendre parce qu'il me fait voir clair en moi, parce qu'il me fait voir au creux de mon cour ce que tu peux être pour moi.

Voici donc ce que m'inspire ma rêverie...

Votre âme est un paysage choisi

(Verlaine)

Laboureur exilé des terres disparues

Je sème ton soleil aux sillons de ma joie

Et ton nom me suffit pour inventer l'aurore

Et ton nom me suffit pour créer un ciel bleu

Pour toi seule les fleurs exhalent leurs parfums

Et toi seule saurais tresser le jonc marin

En la verte couronne docile à ton front

En la verte couronne que nomme le vent

Une mer infinie assoupie sur la plage

Imite de ses flots la couleur de tes yeux

Et le grand tourbillon des oiseaux d'allégresse

Poursuit dans la forêt les échos de ta voix.

Après cette incursion dans le domaine du rêve, je dois m'empresser de revenir au Canada pour ne pas rater la dernière levée de la poste. Mais que les accents volontairement antiques de mon petit poème répandent sur toi et en toi quelques rayons de bonheur...

A bientôt,

Gilbert

P.S. J'ai atteint une moyenne de dix (10) cigarettes par jour!!! Quels progrès! J'espère.que tu ne progresses pas en sens contraire... 
Montréal-Nord, le 20 janvier 1964

Chère Murielle,

J'en ai déjà terminé avec l'examen de ton écriture. Je te soumets les. résultats, les laissant à ton appréciation.

De prime abord, le tout donne une impression d'équilibre et d'harmonie.

Une certaine inégalité dans la grosseur des lettres laisse apercevoir un degré de susceptibilité. Mais ceci est largement compensé par le degré d'inclinaison de ton écriture qui démontre une inclination envers ton entourage.

D'assez nombreux détails indiquent chez toi une tendance à la méfiance et à la prudence. D'autres signes affirment nettement une personne réservée et portée à la réflexion, en même temps qu'une certaine influence du passé.

Voici maintenant une petite remarque un tant soit peu... choquante: les barres de a $t$ " montantes indiquent un esprit de contradiction...

Mais que de belles qualités me laisse voir ta ponctuation! Elle est placée haut sur les mots, signe indiscutable d'un esprit élevé, porté à l'idéalisme et à l'intellectualisme. Même la signature est révélatrice: la tienne dénote la sincérité et la netteté (je te fais remarquer que les "mensonges " bénins que tu aimes bien dire n'ont rien à voir avec la sincérité: ils se classent avec l'enjouement que révèle ton écriture).

Eh voilà ! - c'est fait. Évidemment, n'étant pas un expert en la matière, je me suis contenté d'un examen superficiel, tout en ayant un constant souci de justice et de compréhension. Je me suis contenté de souligner les grandes lignes de ton caractère: qualités et défauts en découlent.

Je suis de ceux qui ne tolèrent pas de demi-mesures. Ce que je hais, je le rejette au complet; ce que j'aime, je l'accepte tout entier...

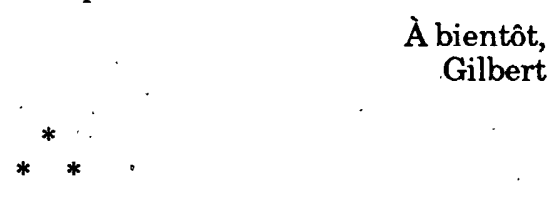

Montréal-Nord, le 28 janvier 1964

Chère Murielle,

Parfois, il est bon de s'arrêter pour jeter un coup d'œil en arrière. Coup d'œil qui permet d'établir des rapports, de dresser une sorte de bilan et de juger une situation.

Avant tout, il est évident que nous avons exercé l'un sur l'autre une influence qui ne peut aller qu'en progressant. De prime abord, nous possédons des caractères, des personnalités, des idéaux qui vont bien ensemble, faute de quoi nous nous serions déplu mutuellement dès notre première rencontre: cela va de soi. Cependant, même avec la meilleure volonté du 
monde, nous ne pouvons empêcher qu'il y ait quelques rugosités, quelques inégalités dans nos caractères; ce sont elles qui créent certaines différences d'opinion qui se soldent par des... « discussions", et c'est tout à fait normal. Et, sur ce point, nous avons gagné beaucoup. Si je puis m'exprimer ainsi, je dirai que le fait de livrer un peu de nos tempéraments pour qu'ils s'adaptent parfaitement l'un à l'autre est un gain énorme pour chacun de nous. Rien n'assouplit le caractère comme cela.

Et que dire du bonheur! Que dire de la douceur de savoir qu'il existe un être qui nous est cher!

Il existe dans la langue française un mot qui surpasse tous les autres par sa noblesse, sa loyauté, sa grandeur et sa simplicité. Sans souffrir de refoulements, je trouve qu'il faut avoir un grand respect pour ce mot, qu'il ne faut le dire ni l'écrire sans discernement; cela tient au caractère surhumain et sacré que je lui prête. Le mot Amour, le mot qui se mesure à la grandeur de l'âme. Se rapportant à toi, je puis l'employer à juste titre; car tu es celle pour qui, sans le savoir, j'avais appris le verbe aimer.

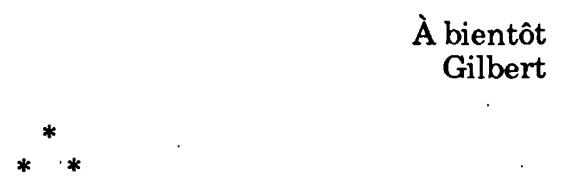

Montréal-Nord, le 10 février 1964

Chère Murielle,

Voici une lettre que tu ne manqueras pas de trouver fort singulière. Ne serait-ce que par le fait qu'elle te parvienne un vendredi; contrairement à mon habitude... Mais enfin, tu conviendras avec moi que ce vendredi est un jour très particulier. Quel meilleur jour pourrais-je choisir pour l'arrivée de ma lettre que celui de la Saint-Valentin?

Comme je le disais plus haut, ce message prend un aspect inhabituel: il sera écrit en trois soirées. C'est si tu veux, une façon de te dédier concrètement, au jour le jour, la pensée qui s'élève vers toi. Ce soir, je voudrais commencer avec une comparaison. Tu as dû remarquer, au cours de ta vie, ces grands oiseaux qu'on appelle les goélands et les mouettes. Avec quelle grâce ils sillonnent l'air! — avec quelle légèreté ils défient les vents! — avec quelle noblesse ils se jouent de l'abîme! Mais qu'un de ces animaux vienne à se poser par terre. Quelle lourdeur dans sa démarche! - comme il éprouve de la difficulté à se mouvoir sur le sol...

On peut faire les mêmes observations sur cette catégorie d'hommes qu'on nomme parfois les poètes. Autant leurs idées se répandent librement sur le papier, autant le fait de les dire vocalement leur est difficile. Je ne t'apprends peut-être pas grand-chose en te révélant cette particularité de mon caractère. Mais sache que ce que je ne dis pas, je le pense. Il est relativement facile d'écrire "je t'aime»: le dire est autre chose, du moins au début. Il me semble cependant que tu sais déjà tout cela, que tu as su comprendre par toi-même cet aspect de ma personnalité. Quoi qu'il en soit, cela ne change rien; mais je trouve qu'il est bon d'en parler quelquefois, par souci de compréhension mutuelle, en signe de confiance. 
Et voilà, tu as suivi avec moi les chemins de ma méditation. C'est tout (jusqu'à demain soir) ... car ma plume montre depuis quelques instants des signes de fatigue... à moins que ce ne soit moi...

Mardi, 11 février 1964

Je viens de découvrir, au cours de certaines recherches dans un gros bouquin, un poème que le hasard m'envoie à propos. Je t'en cite quatre vers... que tu vas reconnaître:

Sans bruit, sur le miroir des lacs profonds et calmes

Le cygne chasse l'onde avec ses larges palmes

Et glisse. Le duvet de ses flancs est pareil

À des neiges d'auril qui croulent au soleil

Tu saisis l'astuce? Depuis tout le temps que tu me répètes ce vers... je puis maintenant t'en' faire connaître l'auteur: Sully Prudhomme. Et il va sans dire que je ne désire faire aucune allusion au papier de toilette «White Swan"... Passons!

Ce soir, je suis dans une forme splendide (ma plume aussi).

Je te présente aujourd'hui un poème d'une allure très moderne. Plus que tout autre, ce poème demande que l'on aille au-delà des mots; au-delà même des idées. Pour bien en saisir toute la portée, il faut lui laisser suggérer librement tout un monde de sentiments, tout le contenu humain qui lui est propre. Lis-le comme tu respirerais le parfum des fleurs, comme tu regarderais le bleu d'un ciel d'été...

\section{La femme indispensable}

Je te prie de me regarder

Pour que la pluie remonte

Toutes ses lignes

Se brisent vers l'horizon

Toutes les vapeurs écloses

Se peignent à rebours

Et la nuit va enfin s'éclairer

Pour te plaire

Pour me plaire

Je te prie de me regarder

Pour m'imprégner

De toute la fixité

Du sphinx de ton sourire

Le sourire de mon aimée

Qui s'épanche vers l'occident

Qui mûrit mes blés en froissant leur soleil

Des spirales gravées

De sa présence 
En m'accrochant
Au rebord d'un rayon
La pierre qui me pétrit
Peut-être je pourrai voir
Peut-être au-delà
Du mystère accueilli
D'un cri d'où tombe la nuit
Je marche au gré de mon regard
La prunelle qui me consume
Qui m'entraîne en avant
Vers le passé
On illumine des fenêtres
Je retraverse des murs
Un cimetière de rẹgards
Je plonge au gré d'une chevelure.

Décidément, je suis incorrigible: il faut que je glisse un poème dans presque chacune de mes lettres. Certains ont de grandes richesses, la beauté et la gloire. Moi, je ne possède que mon cœur à donner et je le donne. Faire le don de soi-même: n'est-ce pas la plus belle définition de l'Amour?

Je cesse d'écrire au moment où les terribles bébés nocturnes* te voient apparaître...

Montréal-Nord, le 24 février 1964

Chère Murielle,

Les mots se montrent parfois impuissants à exprimer ce qui est ressenti au plus profond de l'âme. On cherche une phrase qui saurait traduire exactement ce qui bouillonne dans le cerveau, mais une fois la phrase écrite, on se rend compte que ce qu'elle dit se situe encore bien loin au-dessous de ce qu'on aurait voulu lui dire. Tout cela m'amène à te confier que j'ai quelque peu exagéré, hier, en affirmant que je pouvais prendre une demi-heure pour t'écrire une lettre. $\Pi$ faut t'avouer qu'en moyenne, chaque lettre que je te fais parvenir demande facilement deux heures pour peu que j'y insère un poème. Car tu sais comme moi que, bien qu'il doive être de source spontanée, un poème demande après sa conception un fort travail de finition au point de vue style et langue française. Pour qu'il corresponde le plus possible à la pensée de l'auteur, le poème doit être parfait dans ses moindres détails; chaque mot doit en être pesé. Et d'ailleurs, je t'ai communiqué plusieurs fois mon principe qui veut que je ne te destine que ce que je puis t'offrir de meilleur. Alors, partant de cette façon de penser, il devient naturel que la simple lettre que je t'écris ne doive pas être traitée à la légère, vu qu'elle s'adresse à celle qui est tout un monde à mes yeux.

Voici maintenant que je te présente un petit poème d'un genre que tu connais bien et qu'on pourrait appeler «le genre champêtre ".

* Murielle Ross travaillait dans un hôpital au service de la maternité. 


\section{A perte de vue}

Dans les forêts sans fin que je crée pour toi seule

J'ai gravé dans l'écorce ton nom pour toujours

À jamais j'en ai fait

Le feuillage des saules

Et le parfum discret

De la rose éternelle

J'ai fait de tes cheveux

Le vent des soirs d'été

Et l'Amour que je chante

Au secret de nos cours

Est l'herbe où la rosée de ton âme limpide

Vient reposer sans bruit sa céleste douceur

Je termine én te rappelant que, pour avoir trop parlé, tu es «condamnée» à m'écrire. Mais comme cette a condamnation" me fait plaisir!! Et si cette lettre te coûte quelques efforts, dis-toi bien qu'ils ne seront pas vains, car chacun de tes mots sera pour moi un précieux reflet de ta personne.

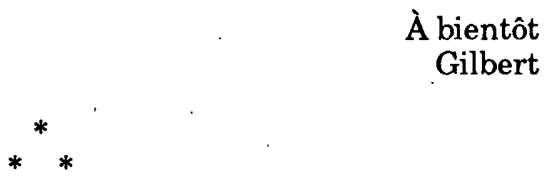

Montréal-Nord, le 9 mars 1964

\section{Chère Murielle,}

Je te demande d'accorder à cette lettre une importance un peu spéciale, car elle souligne un fait décisif, un tournant capital. Lorsque tu connaîtras la nature du sujet que je veux développer ici, tu m'approuveras (je l'espère) de la confiance que je place dans ta grande compréhension.

Je t'ai parlé, dans une lettre assez récente, de la difficulté que j'éprouve à extérioriser vocalement mes sentiments profonds. Chacun a une faiblesse à l'armure de sa personnalité: je t'ai révélé la mienne. Tu te dis peut-être que cela n'est rien, que cela ne représente qu'un infime détail presque sans importance. D'accord, en un sens, j'avoue que c'est un moindre mal. Mais si je regarde la chose d'un point de vue différent, elle prend soudain pour moi un peu plus de relief.

Quand les flots d'un amour immense soulèvent des tempêtes au creux d'un cœur, il arrive un moment où les digues ne peuvent plus contenir la poussée irrésistible de cette marée. Il vient un moment où il faut parler, où il faut ouvrir toutes grandes les portes secrètes de l'âme. Si j'ai pu prononcer hier quelques mots (bien malhabiles, je le concède), qui voulaient t'avouer mon amour, cela représente une victoire. C'est une victoire car il m'a fallu littéralement me vaincre moi-même; car il m'a fallu abattre le mur qui s'élevait entre mon cœur et ma bouche. Et sois assurée que toi seule pouvait opérer cette révolution en moi... Pour terminer, en passant à un sujet plus matériel, je veux te laisser imaginer quelle fut ma grande joie, mon bonheur immense en m'apercevant, au sortir de chez toi, hier, qu'il NEIGEAIT!!!!!!!! 
Ô volupté de patauger dans la neige en simples souliers !!! Tu conçois aisément quelle fut mon allégresse en sentant poindre en moi, chemin faisant, les agréables symptômes de la grippe... Et ce matin, je pus goûter dès mon réveil les incomparables délices d'une gorge enflée et d'un nez obstrué (sans parler d'une gentille douleur musculaire dans les bras, suite logique de mes prouesses athlétiques de la veille).

Mais je suis loin d'être abattu par ces quelques désagréments passagers. Je suis loin de m'en faire pour un mois de mars qui nous apporte de si glaciales surprises. Je conserve en moi le plus beau des printemps: le printemps perpétuel qui porte ton nom.

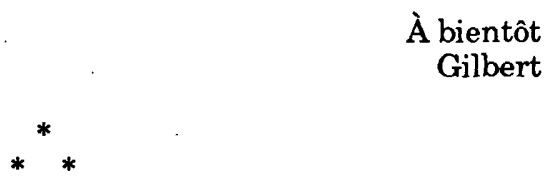

Montréal-Nord, le 31 mars 1964

Chère Murielle,

C'est une impulsion soudaine qui me pousse à t'écrire; un besoin immense de communiquer avec toi, de penser à toi le plus fortement possible. Lorsqu'on est loin de la personne aimée, il me semble normal que l'on souhaite. sa présence. Te dire à quel point tu me manques est une tâche audessus de mes forces et de mes capacités d'expression. Connais-tu cette douce mélancolie éprouvée à la vue, au souvenir même de certains objets? «Voici la place qu'Elle occupa - voici telle ou telle chose qui devient sacrée parce qu'Elle l'a eue en sa possession - voici tel autre objet que Sa main a déplacé et que j'aime voir en cet endroit parce qu'elle l'y a mis... " Une telle façon de penser a toujours été le propre de l'amour. Évidemment, il peut exister quelques variations, selon la personnalité de l'individu en question; mais, en gros, je crois avoir assez bien réussi à exprimer ce que je pense.

Je dois abréger, car le temps... et la poste me pressent. Mais je veux te faire connaître mon dernier poème qui est un hommage au printemps et que je te dédie.

\section{Premier violon}

C'est la chanson d'une aurore blême

Avec le vent dans de lourds cheveux

C'est le rayon qui blondit aux branches

Comme un sourire aux lèvres florales

C'est l'unisson des neiges fondantes

Avec le cour des nuits reniées

Le mausolée des forêts subites

Et le donjon des arbres probables

C'est pour l'argent flottant de la pluie

Que je m'installe au dernier rocher

Et c'est pour l'or prochain des clairières

Que je marie le ciel aux oiseaux. 
«Son sourire me remplissait d'une béatitude infinie; la vibration de. sa voix si douce et cependant fortement timbrée me faisait tressaillir de joie et d'amour.» (Gérard de Nerval)

À bientôt, Gilbert

Montréal-Nord, le 7 avril 1964

Chère Murielle,

Voici ma dix-neuvième lettre... d'après le chiffre que tu m'as mentionné dimanche dernier. Tu vas peut-être te dire que je reviens souvent sur le sujet, mais je me plais encore à répéter quelle est l'importance que j'accorde à ces simples écrits. Tu sais déjà qu'ils sont un chemin sûr vers une intense connaissance mutuelle. Mais as-tu parfois songé au fait que ces lettres donnent à notre amour une dimension infinie dans un monde spirituel. Il est vraiment dommage que, de nos jours, les esprits quelque peu matérialistes négligent ce sublime aspect de l'amour. Comment pourrais-je accepter sans indignation qu'un homme me révèle n'aimer une jeune fille que parce qu'elle est jolie? Quelle mentalité!. comment oublier (ou ignorer) que l'amour ne se limite pas au point de vue physique? Comment ne pas se rendre compte que l'amour véritable doit englober aussi l'esprit et l'âme? Vraiment, je ne puis arriver à comprendre une telle façon de. penser qui se rapproche bien plus de la bête que de l'humain.

Tu sais un peu de quelle façon je conçois ce sentiment noble et généreux entre tous. Pour résumer mon point de vue, il me suffit de dire qu'un être humain se compose d'un corps et d'une âme, donc, il faut aimer les deux ou rien du tout, c'est évident.

Et puis, j’en profite pour te donner mon opinion sur la Beauté. Cette Beauté réside justement dans l'union du matériel et du spirituel. Qu'on ne vienne pas me faire croire qu'un visage qui possède les traits les plus réguliers qui existent, soit Beau si l'âme ne transparaît pas sur ces mêmes traits. Encore plus détestable un visage où ne transparaît qu'une âme vile ou sans profondeur... Au mot Beauté, il faut toujours allier les mots douceur, grâce, harmonie, bonté et étrangeté. Etrangeté parce que le Beau eșt toujours bizarre; parce que pour mériter son nom, la Beauté doit sortir des cadres de l'ordinaire. Une Beauté qui ne serait pas quelque peu bizarre serait par conséquent vulgaire, donc ne pourrait s'appeler Beauté parce que le vulgaire n'est pas beau.

Voici que, comme toujours, je dois me hâter de terminer (la poste!!!), sans avoir eu le temps, malheureusement, d'y insérer mon poème. Mais ce que je t'ai dit aujourd'hui, tu peux quand même le considérer comme de la poésie: parce que je m'adresse, avant tout, à ton cœur bien-aimé.

«Cependant, ses traits n'étaient pas jetés dans ce moule régulier qu'on nous a faussement enseigné à révérer dans les ouvrages classiques du paganisme. Il n'y a pas de beauté exquise, dit lord Verulam, parlant avec 


\section{VOIX \& IMAGES / 45, printemps 1990}

justesse de toutes les formes et de tous les genres de beauté, sans une certaine étrangeté dans lés proportions.» (Edgar Poe)

\section{À bientôt,} Gilbert

Montréal-Nord, le 15 avril 1964

Chère Murielle;

On parle souvent, ici et là, de l'âme sœur. T’es-tu déjà demandé quelle pouvait bien être la signification profonde de ce terme si employé? Pour ma part, je crois en l'existence de cet être qui n'a été créé que pour moi et pour qui j'ai été mis au monde. Tous n'ont pas, comme nous, la chance de reconnaître l'âme qui est faite pour compléter la leur. Combien de gens rencontrent une fois dans leur vie la personne qui leur est destinée... et combien ne savent pas l'identifier! Soit par manque d'intuition, soit par absence de profondeur spirituelle, ils ratent une partie de leur vie.

Lorsqu'on nous a présentés l'un à l'autre pour la première fois, je ne crois pas que l'on se soit connus. Nous nous sommes reconnus. Non pas recionnus par cette mémoire de l'esprit qui garde le souvenir des êtres rencontrés auparavant, mais reconnus par cette mémoire de l'âme qui garde le souvenir d'un idéal et qui sait l'identifier quand il se trouve matérialisé.

Quoi de plus beau qu'un amour qui grandit sans trêve et qui grandira toujours (car l'amour plonge au creux de l'infini) en se fortifiant par le seul fait de son existence... Nous avons su comprendre d'une façon toute naturelle et spontanée que l'amour n'est pas ce que veut faire croire une époque matérialiste. Nous avons su, nous savons et nous saurons que le mot amour est inséparable des mots générosité, affection et compréhension; que nous sommes l'un pour l'autre ce qui s'appelle bonheur dans le sens le plus élevé du mot.

Tout cela pour te bien faire comprendre ce que j'entends par le terme âme-sœur. Et, sans autre transition, je te présente un court poème que j'ai écrit pour illustrer notre existence dans cet autre monde qui n'existe que pour nous.

\section{Double flambeau}

Une mélodie lunaire s'allume

Au brasier profond qui perce les ombres

Et la pâle nuit chantonne en sourdine

Et la pâle nuit danse comme un rire

Une mélodie lunaire s'allume

Aux fruits immédiats des dalles offertes

Et des yeux sont verts à force de croire

Et des yeux sont verts comme un bruit lointain

Une mélodie lunaire s'allume

À l'éclatement soumis au vertige 
DOSSIER 345

Le cri meurt d'amour pour le jour prochain

Le cri meurt d'amour devant son aurore.

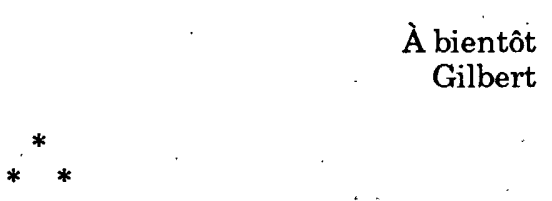

Montréal-Nord, le 20 avril 1964

Chère Murielle,

Tu m'as dit, dimanche, une phrase qui me revient à l'esprit en ce moment. Tu te rappelles cette promenade où notre joie s'est unie au soleil printanier; cette promenade pendant laquelle tu m'as dit que le temps passe terriblement vite lorsque nous sommes ensemble. Combien j'ai constaté, moi aussi, cette fuite rapide des heures, à chacune de nos rencontres. Le bonheur que nous créons par notre mutuelle présence semble faire descendre sur terre une portion d'éternité, un ravissement qui tient du rêve tant l'amour transfigure tout autour de nous. Nous devenons l'un pour l'autre les créateurs d'un monde enchanté où est abolie la notion de temps.

Malheureusement, pour les gens de la poste, cette notion du temps existe bel et bien. Je t'avais prévenue du fait que ma lettre serait quelque peu réduite, vu les circonstances, mais je ne croyais pas devoir l'abréger si considérablement. Mais qu'importe, après tout, la quantité des mots employés: seules comptent vraiment leur vérité, leur tendresse et leur plénitude d'amour.

À bientôt

Gilbert

Montréal-Nord, le 12 mai 1964

Chère Murielle,

Je vois par ma fenêtre le jour qui décline doucement et qui jette-son pastel dans la verdure du jardin. Jamais le printemps ne m'a semblé si beau, si doux; jamais je n'ai ressenti comme à présent la joie qui se dégage de cette saison. C'est que l'amour a placé devant.mes yeux un filtre merveilleux, un filtre à travers lequel tout prend la couleur du rêve. C'est notre printemps à nous seuls, car nous l'avons créé bien avant sa venue matérielle. Nous avons fait fuir le temps étonnamment vite. Ne te semble-t-il pas que notre première rencontre ne date que d'hier? Nous nous sommes connus et, à partir de ce moment, nos pas ont suivi ensemble une route s'écartant de plus en plus de la banalité universelle. Notre chemin se perd dans les nuages et notre soleil ne brûle qu'au feu de notre amour. Nos regards unis se détournent parfois pour contempler, au loin, le grand fleuve de l'humanité. Nous y voyons se débattre des êtres ténébreux, désespérés; des êtres qui n'ont jamais pu 


\section{VOIX \& IMAGES / 45, printemps 1990}

trouver un cœur pour réchauffer leur cœur, une épaule pour y reposer leur front, des yeux pour y voir deviner leurs plus tendres pensées...

Cette courte lettre n'aura été, en somme, qu'un poème. Mais est-il meilleure façon pour un poète de s'exprimer? Le symbole amplifie la réalité et permet à l'esprit de sonder au plus profond de l'âme.

*C'en était fait! le visage de cette femme venait de se réfléchir dans son esprit comme en un miroir familier, de s'y incarner, de s'y reconnaître!... de s'y fixer à tout jamais sous une magie de pensées presque divines! (Villiers de l'Isle-Adam)

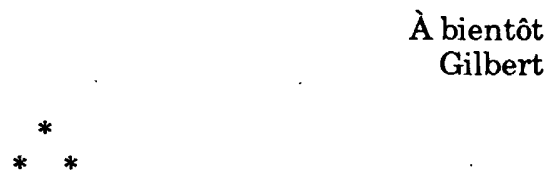

Montréal-Nord, le 19 mai 1964

Chère Murielle,

Que dire après les heures de bonheur que nous avons passées ensemble? Cette fin de semaine ne te semble-t-elle pas un rêve - un long rêve (mais trop court) vécu à deux? C'était vendredi... et, subitement, en un éclair, cè fut lundi - entre ces limites temporelles... le paradis. Je n'aurais jamais cru qu'un être humain puisse ressentir de si grand bonheur ici-bas... mais étions-nous bien sur cette terre? Nos deux cœurs perdus dans une sublime extase d'amour n'ont-ils pas accédé à un monde n'ayant plus rien de commun avec le « monde» des autres?

Souviens-toi, dimanche, quand nous étions assis devant la maison... Tu m'as dit que nous ne ressemblions pas au reste des humains. Comme tu as raison! loin de moi cependant l'idée de mépriser ceux qui ne nous ressemblent pas. Mais il est bon de voir clair en nous et de nous rendre compte de ce que nous sommes vraiment. Nous avons compris que l'amour doit s'abreuver aux sources mêmes de nos personnalités et qu'il doit fondre en- semble des aspirations semblables pour en former un seul et unique idéal notre idéal... un désir commun.

Je vais te dire pourquoi je n'ai pas voulu te révéler la nature de ce fameux. souhait que tu veux tant connaître. Je`n'ai pas voulu te l'avouer pour la simple raison que voici: tu savais, de toi-même, en quoi consistait ce vœu. Quand nous sommes allés voir ces maisons, ces meubles, dimanche, nos âmes se sont ouvertes inconsciemment... On ne dit pas sans raison * notre maison, nos meubles "; on ne fait pas de projets d'avenir comme les nôtres sans être poussés par un amour profond et éternel... J'en ai assez dit - peut-être trop, car tu sais parfaitement tout ce que je viens d'écrire, et tu me diras toi-même si tout le * mystère " est suffisamment éclairci.

Voilà pour l'indice! Pressé que je suis par la hantise de la poste, je dois terminer ici en te disant que tu me manques énormément... plus que je ne saurais dire.

À bientôt

Gilbert 
Montréal, le 2 juin 1964

Chère Murielle,

Je t'écris ce soir en hommage à ta présence que je porte toujours en moi. Tel un nuage, ton image remplit mon esprit, imprègne ma personnalité de tout l'amour qu'elle reflète. Où que je regarde, tout me rappelle celle que j’aime. Les feuilles que le vent agite sont les échos de la voix que je chéris; la lune que voile une dernière vapeur nocturne est la douceur inexprimable que répand le moindre regard de ma bien-aimée; le balancement des herbes endormies ne contient-il pas un peu la tendresse infinie de son sourire?

Je pourrais continuer ainsi indéfiniment, tant tu fais partie de moimême. Mais des paroles pourraient-elles esquisser seulement un pâle aperçu de ce grand amour que nul mot ne saurait exprimer? Parlant de l'amour, Maupassant a dit: «Fort comme la mort.» Moi, je dis: fort comme la vie. C'està-dire qu'il existe pour moi un être que j'aime comme j'aime la vie, comme je bois les rayons du soleil: un être irremplaçable. Si je dis irremplaçable, c'est que l'amour possède cette faculté de faire ressortir ce que la personne aimée peut avoir qui la rend vraiment unique. C'est pourquoi, débordant les cadres de la personnalité, on en vient à aimer même tout ce qui se rapporte directement à celle-ci: on aime ses gestes, le bruit de son pas, sa façon de parler, son parfum... Car l'amour est infini quand il est compris comme nous le comprenons; quand il plonge ses racines au plus profond de l'âme. Un tel amour doit toujours aller en progressant: n'en sommes-nous pas la preuve?

Je viens de t'exposer un autre aspect de ma conception de l'amour, et je crois qu'il trouvera en toi un écho favorable, un point de vue semblable.

En terminant, je te présente un court poème, fruit de la philosophie poétique que je t'ai un peu expliquée la semaine dernière; une poésie où des correspondances mystérieuses s'établissent entre les formes, les couleurs, la musique, là pensée...

\section{Mirages}

Une colonne effrite son marbre

Songeusement sur l'étang blanchi

Le froissement des âmes perdues

Se donne au ciel des airs de corbeaux

Une clochette

Imprègne l'air

Des moiteurs sombres des vieux sabbats

Un vieillard que la nuit fait pleurer

Repose au creux de l'onde immobile

Un cor agite les feuilles pâles

Et voilà! Quatre longs jours encore me séparent de toi : puissent-ils passer au plus vite... puissent les heures s'envoler rapidement pour nous réunir enfin. 


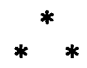

Montréal-Nord, le $1^{\text {er }}$ septembre 1964

Ma biche chérie,

Vraiment, nous fûmes trop choyés durant les deux semaines qui viennent (ô combien vite) de s'écouler. Si tu pouvais savoir à quel point les heures passées loin de toi me semblent vides, ternes, sans signification. Comme il me paraît loin, parfois; le jour tant appelé où nous unirons enfin nos vies et nos destinées... Nous entrerons dans la vie rêvée par la porte des fleurs. À jamais je te ferai de mes bras amoureux un collier de tendresse. Plus que jamais tes yeux, tes yeux tant aimés, étoiles que moi seul vis briller au fond d'une nuit très douce, guideront ma route vers un bonheur inimaginable. Tels maintenant et toujours, les feux farouches de mon esprit réchaufferont ton âme si tendre et si généreuse. Sur le temple arrondi de tes lèvres, je scellerai maint pacte d'éternel amour. Nos amours grandiront, comme le chêne que la vigne enlace, sous l'ombrage parfumé de tes cheveux déployés. Nous ignorerons la frontière abominable qui sépare le rêve du réel...

C'est ainsi que je vois (et ainsi ce sera) notre prochaine vie commune. Cette lettre marque à peu près l'anniversaire du premier message que je t'ai écrit, il me semble, vers la même époque l'an dernier. Entre cette première lettre et celle-ci, il y a un monde: n'est-ce pas la preuve évidente que l'amour doive croître progressivement, comme la plus belle des fleurs, et non pas éclater soudainement, sans raison, comme un feu de paille? L'amour tel que nous le connaissons me fait songer à un brasier intensément ardent que nous alimentons sans cesse, de sorte que sa flamme s'enfle et brûle avec plus de vigueur chaque jour. Nous pouvons nous montrer fiers d'un semblable amour et le laisser éclater sur nos visages: il nous appartient et s'intègre à la singularité même de nos personnalités.

Je dois malheureusement terminer ici cette lettre; mais pas sans t'avoir embrassée avec ferveur, d'un baiser qui se plonge déjà dans le futur paradisiaque de notre prochaine rencontre.

À bientôt (je t'aime et je t'aime) Gilbert

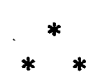

Montréal-Nord, le 7 octobre 1964

Mon ange chéri,

Voici, en guise de plaque commémorative à la fois d'un anniversaire et de fiançailles, plus d'un papier empreint d'un suprême amour. In serait banal de te souhaiter ici les vœux habituels à ces circonstances. Je veux célébrer ton nouvel âge comme on respire une fleur - une fleur où s'épanouissent vingt-deux pétales que, l'un après l'autre, je baise en murmurant, comme en un rêve fou...

Considérant la signification réelle de ce jour de bonheur, j’ai cru opportun de te présenter un petit poème que l'Amour seul m'inspira. Plus que jamais 
dans mes vers, j'ai mis toute mon âme en ceux-ci qui évoquent notre future nuit nuptiale. Je ferai suivre ce poème d'une explication détaillée: car l'éblouissement d'un style travaillé ainsi qu'un diamant amoureux peut dissimuler un peu la pensée qui lui sert de fond.

\section{Accomplissement}

Une lisse pâleur d'aile moite amplement

Déployée sitôt chu l'or d'un nuptial soir

En l'effluve jaillie noire que nul métal

N'opprime veut s'offrir qu'un rien de gaze voile

En frisson vespéral que le soupçon n'altère

D'albâtre réitère un sein d'ange endormi

La douceur son soupir proférant orchestral

D'intemporel rubis qui de neige s'enivre

D'un rêve de jadis mué tangiblement

L'abandon chastement licite s'éblouit

À la nubilité languide que tel lys

Eternel perpétue dans maint songe insolite.

\section{En d'autres mots:}

la femme de mes rêves, telle une blanche aile d'oiseau, est étendue mollement au milieu de ses cheveux noirs, libres, que nulle broche ne retient plus; rencontre d'un vêtement translucide (de la gaze), elle veut s'offrir à son mari quand s'est obscurci le ciel d'or du ciel nuptial.

avec un frisson, sentant le bonheur prochain, sans crainte et sans fausse pruderie, la douceur expose un sein d'ange endormi, tout en laissant échapper de ses lèvres (le rubis sur la neige des dents) un soupir mélodieux, orchestral.

un rêve osé jadis se réalise enfin: Elle est là, s'abandonnant chastement, vu que cela est devenu licite, permis; cette nouvelle épousée, comme un lys, issue d'un rêve, suscitera encore maint songe de poésie.

Voilà, je crois, un des plus beaux hommages que je pouvais faire à ta féminité sublimée. Si tu relis maintenant le poème, tu pourras, connaissant la pensée qui le conduit, y voir se cristalliser en un infini de beauté, mes rêves éperdus...

À bientôt

Gilbert

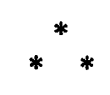

Montréal-Nord, le 2 novembre 1964

Ma fiancée chérie,

Il faut considérer cette lettre comme un cri d'amour: un cri que je lance vers toi, avec toute la force de ce cœur qui t'appartient tout entier. Si 
seulement je pouvais trouver les mots - le Mot - qui, plein de toute ma tendresse, saurait t'exprimer ce que je ressens pour toi. Je voudrais, de chacune de mes paroles, t'entourer de tout le vertige de cet amour brûlant comme un soleil de bonheur...

J'écoute, en ce moment, une musique pleine d'extase et je songe à ces heureux moments, dans le futur où nous entendrons ces mêmes notes, ensemble, dans notre foyer poétique. Ce pourrait bien être l'une de ces voluptueuses soirées d'hiver dont nous avons tant parlé: : nous serons assis dans la bibliothèque, en la chaleur de notre mutuel amour, tandis que, dehors, quelque vent glacé viendra flageller, de flocons poudreux, notre fenêtre. Nous nous regarderons longuement, avec une tendresse presque surhumaine, et notre sourire réciproque contiendra tout ce que nos paroles ne sauront jamais dire. Parfois, nos têtes rapprochées, entremêlant nos cheveux, nous porterons nos regards vers quelque reflet que le rouge d'une lampe attachera, dans la pénombre, à une tenture mollement disposée, antiquement, sur une tapisserie d'autrefois. Ce sera en de telles soirées que nous sentirons monter. en nous une ardeur d'aimer impossible à imaginer et à décrire; ce sera en de telles soirées qu'un délire commun unira nos cœurs et nos corps, dans les effluves d'une musique qu'entrecouperont nos soupirs éperdus...

Aurions-nous pu rêver mieux que la très future réalité où nous vivrons? Ce sera, crois-moi, un rêve éveillé, un paradis sur cette terre, créés par le seul fait que nous soyons ensemble et que nous nous aimions.

Mais le facteur, lui, ne rêve pas et cet important (pour la circonstance) personnage, je sais qu'il se hâte, le pervers, vers la boîte aux lettres. Alors, je n'ai que le temps de te faire parvenir, ci-inclus, un beau baiser.

\section{À bientôt, mon amour,}

Gilbert

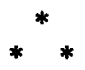

Montréal-Nord, le 11 janvier 1965

Chère Murielle,

En ce moment, tu te prépares, sans doute, pour cet affreux travail nocturne (il est près de 11 heures P.M.). Que de souvenirs! Je me rappelle ces temps où nous restions, en moyenne, quatre ou cinq jours sans nous voir. Tu te souviens? C'était d'ailleurs un des buts de mes lettres, de combler ce vide en nous, laissé par une absence. Ma toute première lettre, elle-même, toute timide, poussait déjà vers toi un délirant cri d'amour. Je t'avais déjà défini ces messages comme des conversations où j'avançais des idées auxquelles, en ta belle âme, tu répondais. Rien de changé à tout cela, mon amour ! Je charge toujours, de plus en plus, mes lettres d'un potentiel d'infini définissant notre amour.

Oui! ce soir, je me sens, comme autrefois, plein de ce suprême bonheur de marier étroitement nos esprits. Ce doit, jamais nous ne l'oublierons, toujours être l'Esprit qui conduise notre vie à deux. Voilà pourquoi nous ne cesserons pas une seule fois de planer en la joie idéale d'être deux à penser ensemble: nos corps vieilliront, mais notre Monde intérieur, lui, n'aura pas à 
subir les outrages du temps. Quand la violence de notre jeune ardeur de s'aimer en nos corps' s'apaisera, nous goûterons alors le Nectar que nous aurons su préparer; nous vivrons, plus près que jamais l'un de l'autre, le plus parfait, le plus sublime des amours: l'Amour que nous garderons pour toute l'éternité, car l'Esprit ne meurt pas.

Comme tu le vois, ma Chérie, nous avons encore un long chemin à parcourir, tous les deux... et un jour, accompagnés de nos enfants, nous ressentirons, dans toute sa plénitude - combien nous le bénirons! l'incomparable bonheur de se comprendre...

Une signature, maintenant, doit venir clore cet entretien: que le nom exprimé en ces caractères s'imprègne en ton cœur aimant, et qu'il y apporte tout l'amour que j'y veux déverser.

Je t'embrasse et je t'aime.

À bientôt,

Gilbert

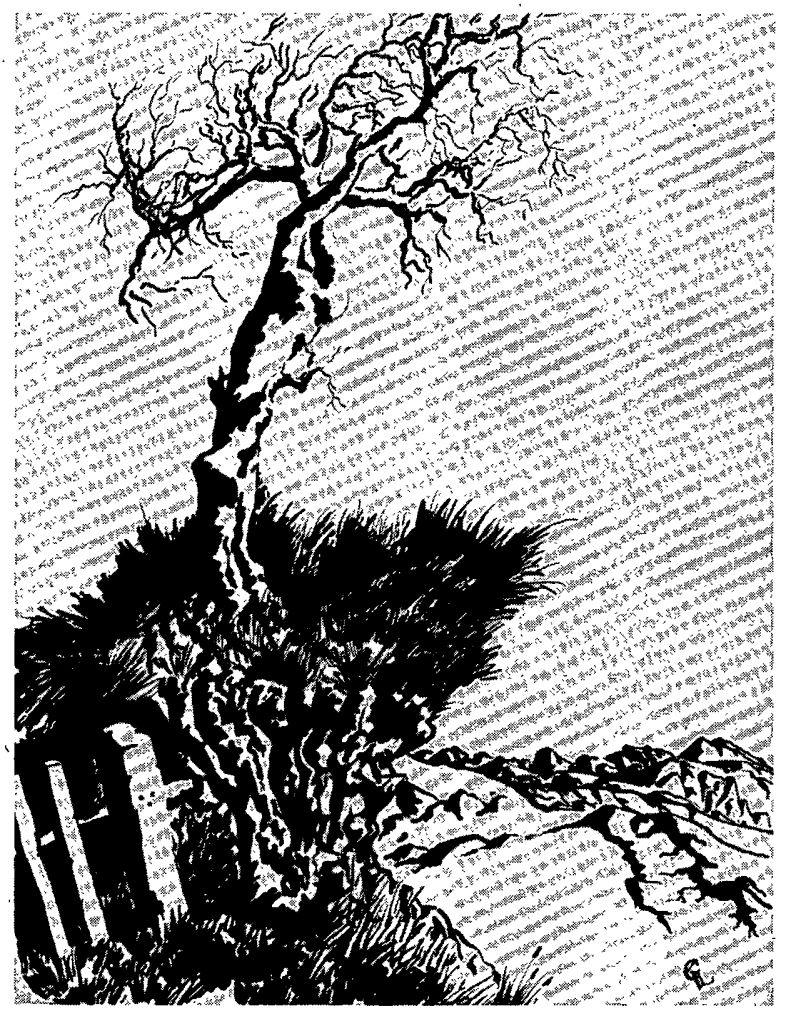

Gilbert La Rocque, encre sur papier 